\title{
Opportunities and challenges for self-monitoring technologies for healthy aging: An in-situ study
}

\author{
Mirana Randriambelonoro MSc ${ }^{\mathrm{a}, *}$ \\ Yu Chen $\mathrm{PhD}^{\mathrm{b}}$ \\ Onur Yuruten $\mathrm{PhD}^{\mathrm{c}}$ \\ Pearl Pu PhD ${ }^{\mathrm{C}}$
}

\begin{abstract}
aDepartment of Radiology and Medical Informatics, University of Geneva, Geneva, Switzerland; ${ }^{b}$ Department of Informatics, University of California Irvine, Irvine, United States; ' ${ }^{\text {Human }}$ Computer Interaction Lab, Swiss Federal Institute of Technology in Lausanne, Lausanne, Switzerland; *Corresponding author: mirana.randriambelonoro@etu.unige.ch
\end{abstract}

\begin{abstract}
M. Randriambelonro, Y. Chen, O. Yuruten, P. Pu. Opportunities and challenges for self-monitoring technologies for health aging: An in-situ study. Gerontechnology 2017; 16(3):173180; https://doi.org/10.4017/gt.2017.16.3.006.00 The current need to adopt a healthy lifestyle opens the way for researchers to investigate the efficiency of using technology to motivate a change towards such behavior. Senior people's technology adoption barriers have been largely investigated in previous research but remain unclear to design efficient solution to promote behavior change in the long term. In this paper, we present the findings of an experiment with 20 older adults using pervasive sensors for 6 weeks at their homes. In particular, we present their attitudes for physical activities and self-monitoring technologies before and after the actual usage. We then discuss the opportunities and challenges for designing self-monitoring technologies for healthy aging.
\end{abstract}

Keywords: Health, behavior change, activity monitoring, qualitative studies, older adults, physical activity

Faced with the constant growth of aging population, the need to promote an environment for healthy aging is expanding ${ }^{1-3}$. Although maintaining healthy behavior has been shown to be highly beneficial for older adults' health and wellbeing, the challenge remains in motivating the adoption and the long-term engagement in such behavior ${ }^{4}$. There are opportunities for emerging technology to increase older adults' engagement in being physically active and managing their health. Within the European REACH (Responsive Engagement of the Elderly promoting Activity and Customized Healthcare) project ${ }^{5}$, the goal is to learn the older adults' behavior by collecting physical activity and health related data in order to provide personalized health recommendation to them. For this purpose, we conducted an ethnographic study for data collection to get insights on older adults' readiness, willingness, and challenges to adopt pervasive sensors and applications for healthy ageing.

The goal of this ethnographic study is three folded: First, we will obtain insights on older adults' attitudes towards increasing physical activity as well as their readiness towards tracking technologies. Second, we will identify senior's potential behavior changes as well as their usage intention. Third, we will shed light on the opportuni- ties and barriers for them to be monitored and try to understand how they would integrate the system in their daily life.

We present here the findings of a 6-week study with 20 senior people using health monitoring sensors and applications in their home environment. We discuss senior individuals' readiness, willingness and challenges to accept such technology to manage their health. We then discuss their behavior change and aptitude for sustained usage at the end of the study.

\section{RELATED WORKS}

A sufficient amount of moderate intensity physical activities (150 minutes) have been shown to have a substantial impact on older adults' (age $>65$ ) health ${ }^{6}$, which would result in a stronger independence and a higher quality of life ${ }^{7}$. However, the vast majority of older population has difficulty in meeting this recommended lifestyle ${ }^{8}$.

Meanwhile, technologies for behavior change to promote healthy living are widely investigated in the research community, ${ }^{4,}$. Researchers tested different technologies including pervasive sensors $^{10}$ and mobile applications ${ }^{11-13}$ to help people track their health data. A rich body of work also leveraged goal-setting, rewards, and metaphors 
to motivate healthy behavior change, such as promoting physical activities.

For senior adults, in particular, Schrack et al., ${ }^{10}$ explored the efficiency of several wearable activity monitors, and Vichitvanichphong et al., ${ }^{14}$ focused on the older adults' perceived value of assistive technologies. Several studies ${ }^{15-17}$ using pervasive sensors as intervention showed promising results in terms of health awareness and small improvement in exercise levels, but no consistent changes have been observed. Consequently, despite these ongoing works on older adults' adoption of persuasive technology for behavior change, the barriers to senior's acceptance of these systems still need further investigations. The purpose of this study is an attempt to give more insights on older adults' opportunities and challenges in adopting such technologies for designers and engineers to propose personalized and adapted interventions that will induce steady changes in older adults' daily life.

\section{Methodology \\ Participants}

We recruited 20 older adult participants from a daily care home, a primary healthcare center and a main hospital in a large city in Switzerland. As we ensured the anonymity of participants' data, we were exempted to go through the institution's Ethical Committee. Our inclusion criteria were: $65+$ years old, living at home, and in need of occasional help for their daily activities. The participants include 13 females and 7 males; among them 3 couples took part in the study together. Their ages range between 65 and 89, with a mean age of 77. 6 participants live alone and the rest live with their spouses. Only a few of them still have occasional social activities. 8 of them have serious health conditions limiting them in doing physical activities. All of them have a TV and watch it or use it as background noise on purpose. Most of them use landline phones for communication. 8 of them have smartphones, and among them, four use social media applications (WhatsApp) motivated by their grandchildren; one of them uses a pedometer application introduced by her daughter and 1 uses a photo editing app. 9 participants have tablets and use them mainly for watching news, searching on internet and listening to music. 2 of them had experience using pedometers before the study but they stopped using it after a year due to device failure.

We choose to include 20 participants to make sure our findings come to theoretical saturation. In a qualitative study, saturation is reached when additional participants do not provide additional insights. Guest et al., ${ }^{18}$, Crouch et al., ${ }^{19}$, and Strauss $^{20}$ suggests that saturation occurs around
12 participants if the group is homogeneous. A minimum of 15 participants is then necessary to ensure saturation. In line with other qualitative studies related to our subject ${ }^{21-23}$, we decided to include 20 participants.

\section{Materials and incentives}

We provided each participant with a fitness tracker and a smart scale. For fitness tracker, we chose Fitbit Charge 2 tracker (https://www.fitbit. com/charge2). For smart scales, half of the participants received the Fitbit Aria scale (https:// www.fitbit.com/aria) and the other half the Withings Body Cardio scale (https://www.withings. com/ch/en/products/body-cardio). We selected our sensor through a comparison of products based on a number of criteria from Yumak et al., survey ${ }^{9}$. These criteria include safety certifications, measurement accuracies, ease of use (wearability, battery life, data storage, ease of use of the software interface), usefulness (the range of information collected via the sensor), seamlessness of data transmission, data accessibility (using public API, manual download), and affordability-see Table 1 and Table 2 for the summary. Participants received the tracker and the scale as incentives at the end the study.

\section{Procedure}

We conducted a qualitative study with participants who used the sensors at their homes for 6 weeks. Each participant went through two main phases: the setup phase and the experiment phase (Figure 1). At the beginning of the setup phase, in-between the 2 phases and at the end of the experiment phase, we met the participants for individual interviews.

During the setup phase, the participants received the devices and familiarized them for 2 weeks. The goal of this phase is for them to get familiar with wearing and using the sensors and for the study conductors to identify any potential difficulties.

During the experiment phase, we randomly split the participants into two groups. The first group continued to use the devices for 4 weeks. For the second group, in addition to continuing to use the devices for 4 weeks, they also received visualizations about their activity data.

We then conducted 2 semi-structured interviews (at the beginning and at the end) and an intermediate debriefing (in-between) with each participant. We prepared the topics of interview questions by referring to the ethnographic methodologies by Sabelli et al., ${ }^{24}$ and requirements for lifestyle management systems by Yumak et al., ${ }^{9} \mathrm{We}$ encouraged the users to share their experience and to show us how they used the devices at their 
Table 1. The summary of our sensor evaluations. Full-black circles, partially full circles, and white circles indicate high, acceptable, and low scores respectively

\begin{tabular}{l} 
Device name \\
\hline Wahoo Tickr $\mathrm{X}$ \\
\hline Sunnto Smart \\
Sensor \\
Fitbit Charge 2 \\
Sitbit Onark3 Cardio \\
Mio Alpha 2
\end{tabular}

own willingness. The interviews were conducted at the participant's home in French, which is the participants' native language. All the interviews were audio-recorded. We first debriefed them about the study and obtained their consents.

The pre-study semi-structured interview lasted around 60minutes. The goal was to understand participants' daily life, health condition and physical activities. We also aimed to know their technology usage and their expectation about technologies for health management and physical activity monitoring.

The intermediate debriefing lasted 15 minutes. The goal was to make sure that the participants could use the devices correctly and integrate them in their daily life. We started to get few feedbacks after a first usage. We then started the experiment phase and helped half of the participant visualize their activity and health parameters on the Fitbit application and the Withings application.

The post-study interview lasted around 45 minutes. The goal of this interview was to get user's feedback after interacting with the devices and to understand their acceptance and adoption of the tools, as well as the challenges they faced during the experiment.

\section{Data analysis}

We audio-recorded all interviews (total of 2,247 min), transcribed them into English, and analyzed the resulting 307,839 words according to the grounded-theory approach. After reading all the transcripts, we generated a starting list of codes based on the research questions and the patterns that emerged from the data. We then returned to the data and conducted a systematic axial coding to identify emergent themes. After several coding iterations, we identified and categorized the themes and reached a consensus.

\section{FindinGS}

In this section, we focus on the older adult people's perception on activity tracking devices before and after usage. Results on their attitudes on scales and visualization will be presented in another paper.

As our primary purpose in this paper is not to quantitatively measure the intervention effectiveness but rather to elicit users' experience in using self-monitoring technologies for healthy aging, we do not have a control group. However, the evolution of the participants' number of steps during the experiment showed an increase of 1609 steps. We compared the mean number of steps in Week 1 (4573) and the mean number of steps in Week 6 (6182).

\section{Before usage}

Attitudes towards increasing physical activities Regarding older adults' opinion about physical activity, different points of view emerged from the discussion. Most of them $(n=14)$ were aware of the benefits of doing physical exercise for their health but failed to do so due to inability and lack of motivation. P2 said: "I know it is good but I am not really motivated to walk when I am alone. When my friend was there I was more motivated but now she is sick". P10 mentioned: "I really like walking but with my leg I cannot go far anymore, it is really painful so I stay home. When I am bored at home, I try to go out a bit but only to take coffee and cake downstairs, not far away". 
Table 2. The summary of our intelligent scale evaluations. Full-black circles, partially full circles, and white circles indicate high, acceptable, and low scores respectively

\begin{tabular}{lll} 
Device name & Accuracy & Safety \\
\hline Fitbit Aria
\end{tabular}

Two participants had been very active in sport in their younger age, but they no longer felt the need to continue anymore. P16 said: "You know I did a lot of football before, during 30 years of my life. Now I think I have done enough, it's not for my age anymore. I just watch sport on TV". One participant who is very healthy doesn't feel the need to do any physical exercise. He said: "My doctor told me to walk 3 times a week but I don't do it, because I don't feel like I need it. I don't have any condition, I am very healthy. I am not the type to go walk just for the pleasure to walk, there should be a purpose besides".

\section{Readiness towards tracking technologies}

Here, we attempt to understand older adults' readiness to adopt such devices and integrate them in their daily life. Nowadays, technological solution is not always adapted to senior's needs and ability, which then creates a disinterest on their side ${ }^{25}$. However, some of our participants $(n=4)$ were eager to try new tools and devices. P4 told us: "I am very interested in technology. It's a pity that I don't have the ability to use more complex things like computer but my children offered me a tablet and I learned by myself how to use it. I spend several nights trying to understand how it works and now I manage to use it". P15 often spends his afternoon trying to help his friends doing things on his computer. He reported: "As I am very interested in computer, he calls me when he has a problem so we try to solve it together".

Five participants got interest in diverse mobile applications introduced by their family or friends. P7 proudly told us: "let me show what I can do with my smartphone, I know how to use WhatsApp, you know, and I even edit my photo before sending it to my grandchildren". P3 said: "my daughter got me interested in this health application on the phone that can count steps, so I try to do 6000 steps every day".

One participant who was not interested in technology at all found the devices we gave them manageable and acceptable to use. P5 said: "I am not very a technological person, I don't have computer, neither a smartphone, it is too complicated. I just have a fixed phone and a mobile phone that
I barely manage to use. I am a bit afraid, but maybe I can manage to learn it if it is simple."

Before using the devices, our intent was to understand older adults' attitudes towards increasing physical activity as well as their readiness towards tracking technologies. The main barriers of being physically active were the absence of motivation, the incapacity due to health condition and the lack of perceived usefulness of physical exercise. Furthermore, although half of the participants were not technology oriented, personal interest and enthusiasm driven by family towards technology were observed.

\section{After usage}

For post-usage, two participants (P13 and P20) couldn't have been reached due to the wife hospitalization, so we analyzed the data based on 18 participants.

\section{Behavioral change}

After 6 weeks' usage, 10 participants reported to us a positive change on their behavior in their everyday life. They replaced some of their sedentary activities such as reading, knitting or writing letters to a friend to going outside and walking in their neighborhood. P5 shared: "I feel like I don't have time to do my little things anymore, because I go out to walk every day. I realize it takes me a lot of time to do it, but I like doing it. So, I stay up late at night to catch up with the things I didn't finish".

Even though we didn't purposely ask them to reach a specific goal, most of them fixed a personal objective themselves and acted accordingly in order to reach it. P1 said: "I make sure I do at least 4000 steps every day, so I go out and walk more since I have the watch". P4 who is very motivated even walk at home to increase her number of steps. She told us: "Now, I even do the exercise the physiotherapist asked me to do, and I look at the watch to know how much I have done". Some participants didn't walk much more but tried to take more stairs. P15 said: "My physiotherapist always told me that I should do at least 2 floors by stairs as I live on the $4^{\text {th }}$ floor, but now I always take the stairs to come up here. I feel like I have less difficulty to breath while I do that." 


\section{S elf - mo n it o ring te ch nollog i es}

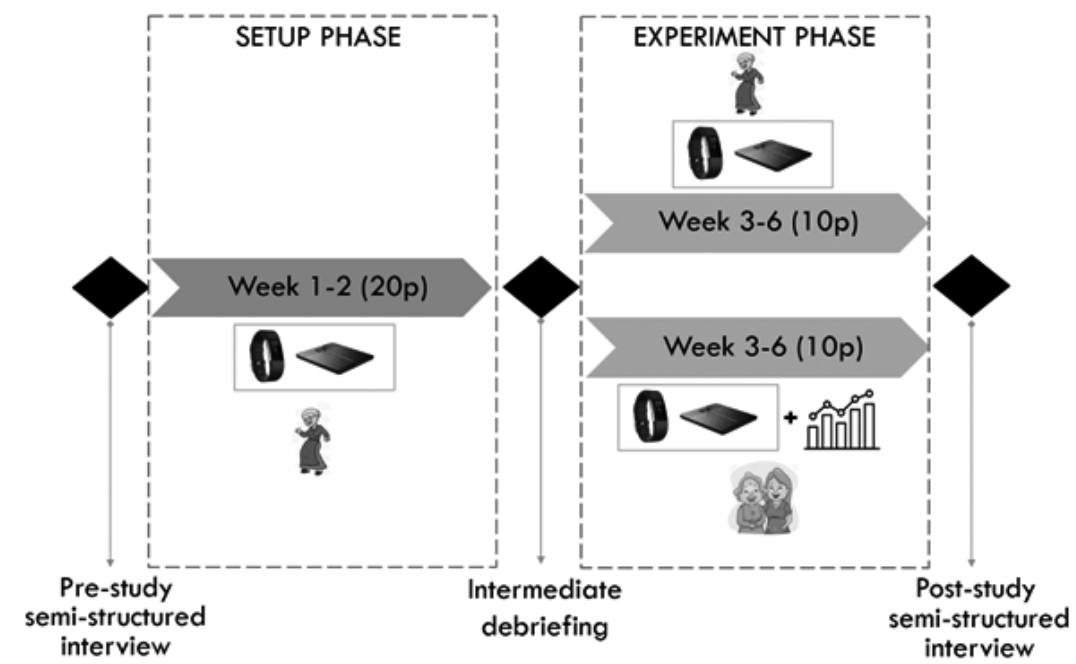

Figure 1. Setup phase and experiment phase of the study

Participants were motivated to continue using the device because it was engaging, fun and allowed them to set personal goal and track their improvement. In total 15 participants kept the devices. Some of them (P5, P14, P17) even started to make plans and future goals with the tools. P5 ensured she will be able to charge it anywhere because she is planning to go on holiday in a few months. P14 wanted to know

For P17, even though he was on a wheelchair, wearing the watch motivated him to move around. He said: "now I am going in circle inside the house instead of sleeping in my wheelchair while waiting for the nurse to come". His wife reported to us: "as he cannot see what is displayed on the watch, he always bugs me to check how much steps he has done". P17 also became very dependent on the device. His wife contacted us after the watch stopped working: "the watch didn't work for 3 days now, and my husband is sleeping a lot again. He really insisted that I contact you so that you can solve the problem because he really needs it as a motivation".

Meanwhile, five participants reported that no changes had occurred in their daily life. They were doing the same activities as before and discovered that they could reach a certain number steps or stairs by going around at home and having their daily routine. P19 said: "I can't say I changed my habits due to the watch but with what I do every day I could reach around 7000 steps. This is what I can do, I don't think I can do more and the watch wouldn't change that". P7 also reported: "I don't feel that I am walking more but as I live in a house with stairs, I have a proof now that I am doing a lot of activity by going up and down".

\section{Usage Intention}

We investigated usage intention by looking at whether participants had the intention to continue using the device. Overall, 15 out of 18 participants expressed willingness to continue using the device, while 3 out of 18 participants did not plan to continue using it. more about making a food plan through the application because he would like to lose weight. P17 who is half-paralyzed told us: "Now I do at least 2000 steps a day but my goal is to reach at least 3000 steps in summer".

We were surprised by P5 behavior. As she didn't know how to use a computer, she took the time to note every parameter in a notebook for every day of the experiment, without us asking her to do that. She started to self-reflect on her personal data and asked us to explain some parameters she couldn't understand. She was ready with a list of questions about how to use either a functionality of the devices either a data she couldn't understand. She said: "I liked it because it makes me have a goal and walk more. Someone told me that since the study is over now, I will have more time for other activities. But I told her that even if the study finished, I will continue to walk and record everything because I like it".

Although some of the participants $(n=5)$ were a bit skeptical about the efficiency of the technological solution at the beginning and wanted to return the device to us at the end of the study, 4 of them changed their mind. P16 said: "it gives interesting information and it doesn't require so much effort from me, so I will keep it". P11 reported: "at first when you came, I thought what is this thing again, but you now, I find it very funny, I get used to the little message. It's like a game. If you told me you would take it now, I would feel that something is missing".

However, some participants did not want to use the devices anymore because of perceived inutility, unwanted behavior related to the tools, and 
fear of the equipment negatively impacting their health. For example, P12 who has a lot of difficulty in walking gave us back the device at the end the study. She said: "I knew from the beginning that it wouldn't change anything in my daily life. My problem is really that I can't do much and it is not a technology that would tell me what I have to do". Another participant (P2) also returned the watch but kept the scale. She didn't like the fact that she wanted to look at the watch all the time to see how much she has done. One participant (P9) dropped the study at the end of the setup phase. Due to a health problem that occurred during the experience, she preferred to stop using the devices, fearing that there would be a link to that.

\section{Discussion}

Our findings shed light on the older adults' readiness, experience, and challenges for adopting technologies for healthy aging.

\section{Opportunities}

Our participants had different reasons to participate in the experience. 10 thought the experience would be interesting, 6 participated due to their trust in the institution which proposed the project and 4 said they accepted to take part in the study only to help the investigator. However, in the end 15 out of 18 kept the devices and had the intention to continue using them. While designing augmented reality game for older adults, Laine et al., ${ }^{26}$ also observed hesitation in their participants at first, but discovered later that the team competitiveness succeeded to engage them in doing the physical exercise through the game. In our case, senior's usage intention and willingness to integrate the devices in their daily life could be explained by the systems simplicity, practicality, and the possibility to customize it to their needs and ability.

Participants also demonstrated a stronger motivation when the device usage was linked to a personal goal they set. The usefulness takes an important role in user willingness to adopt such devices. Designers of systems that encourage being physically active should consider emphasizing the added value of the technology usage. This could be done by offering timely feedback on the older adults' progress and giving easy to understand correlation between their daily activity and the variation of their personal parameters to allow a self-reflection on their behavior.

In addition, the devices really impacted some user's behavior and induced a relation of dependence. As the seniors broke their old habits to integrate a new system in their daily life, once getting used to it, it became a part of their lifestyle. They considered the device as a companion, a buddy that shows interesting information without constraining them. The need for timely information became greater as well as the need for receiving message that makes them less lonely. Considering this inter-relational aspect would benefit researchers who would want to increase long term engagement in technology usage for behavior change.

\section{Challenges}

Half of our participants showed interest and began to change little things in their behavior. Five participants didn't have any changes in their daily routine but used the tools only as a source of information. However, five participants still found it challenging to integrate in their daily life. The main reasons were linked to the fear of introducing novelty and breaking their old habits, the fear to be dependent of the tools, and the need of a human presence interacting with them.

The fear of novelty, introducing new things and breaking their daily routine remained a challenge for some of our participants. As people get older, they start to create a daily routine to facilitate they everyday life. In addition, although seniors are now more exposed to new technology, their acceptance of it is still difficult to achieve, mainly because of lack of adaptation of these systems to their age-related condition ${ }^{27}$ and their lack of confidence in using the tools correctly. Considering these aspects, it becomes challenging to lead the older adults into creating new habits by inserting new unfamiliar elements in their lifestyle.

One important aspect addressed was also the fear to be dependent of the tools. While some older adult people needs to rely on something due to loneliness and the need of interaction and assistance, others do not find this relation of dependence acceptable. The sensation of being able to control the systems they are using is also very important to them. Engineers should propose a system that allows the older adults to turn it off when necessary, so that they would not feel obliged to look at it every time. Furthermore, designers should focus on positive improvement to avoid senior's culpability, which will decrease their fear of being dependent of the systems.

Finally, the last challenge we observed were linked to the need of social interaction. Although they found the tools interesting, most of them still value the interaction with others. One important aspect that researchers should take into account while designing intervention to encourage physical activity, is to involve real meeting with family, friends and peers through technology. Talking to people, commenting on the news around a coffee or discovering together a new neighborhood would be an efficient motivation for seniors to go out of their home. 


\section{Conclusion}

We conducted an ethnographic study for 6 weeks with 20 senior people at their home. The ultimate goal of this project is to understand whether the older adults would accept to be monitored and whether they would adopt and integrate such tools in their daily life. Although our procedure involved different sensors and setups, we mainly report in this paper older adult people's perception on activity tracking devices before and after usage. After 6 weeks of usage, we discovered changes in behavior and usage intention which allowed us to identify opportunities and challenges for the older adults to adopt sensors and application for health and activity management. This study showed the potential of acceptance and adoption of simple and manageable technology for behavior change. As for us-

\section{Acknowledgements}

We are grateful to the anonymous reviewers for their valuable feedback. We also thank Samira Hammoudi Bouchez and Marie-Luce Guillermin Spahr for helping us recruit the participant. Finally, we thank all the participants for their time and their involvement in the study. This research has received funding from the European Union's Horizon 2020 research and innovation program under grant agreement No 690425.

\section{References}

1. Dishman E. Inventing wellness systems for aging in place. Computer, 2004;37(5):34-41; https://doi. org/10.1109/MC.2004.1297237

2. Ying AHP. Creating a supportive environment for elderly with chronic illness. Plenary session of the Healthy Ageing Convention 2001

3. Wood A, Stankovic J, Virone G, Selavo L, He Z, Cao Q, Doan T, Wu Y, Fang L, Stoleru R. Context-Aware Wireless Sensor Networks for Assisted-Living and Residential Monitoring. IEEE Network 2008;22(4); https://doi.org/10.1109/ MNET.2008.4579768

4. Araullo J, Potter LE. Promoting Physical Activity in Seniors: Future Opportunities with Emerging Technologies. Proceedings of the 2016 ACM SIGMIS Conference on Computers and People Research 2016; pp 57-64; https://doi. org/10.1145/2890602.2890616

5. Responsive Engagement of the Elderly promoting Activity and Customized Healthcare; http:// reach2020.eu; retrieved May 30 ${ }^{\text {th }}, 2017$

6. Centers for Disease Control and Prevention, US Department of Health and Human Services. How much physical activity to older adults need? https://www.cdc.gov/physicalactivity/basics/older_ adults/index.htm; retrieved June $07^{\text {th }}, 2017$

7. Sims J, Hill K, Hunt S, Haralambous B. Physical activity recommendations for older Australians. Australasian Journal on Ageing 2010;29(2):81-87; https://doi.org/10.1111/j.1741-6612.2009.00388.x

8. Prohaska T, Belansky E, Belza B, Buchner D, Marshall V, McTigue K, Wilcox S. Physical activ- age intention, some older adults start to be eager to learn new tools as long as they are able to do it, which is more and more influenced by family from younger generation. However, designers and engineers should consider older adults' fear introducing novelty in their daily life as well as their need for social interaction and their need to remain in control of any system given to them.

So far, this study focused mostly on the qualitative aspect, and particularly on physical activity tracking. In the future, we would focus on the impact of the scales and the data visualization presented in the procedure, go deeper in analyzing quantitatively the participants' data and identify potential guidelines for designing pervasive technology for healthy aging.

ity, public health, and aging: Critical issues and research priorities. The Journals of Gerontology Series B: Psychological Sciences and Social Sciences 2006;61(5):267-273; https://doi.org/10.1093/ geronb/61.5.S267

9. Yumak Z, Pu P. Survey of Sensor-Based Personal Wellness Management Systems. BioNanoScience 2013;3(3):254-269; https://doi.org/10.1007/ s12668-013-0099-0

10. Schrack JA, Cooper R, Koster A, Shiroma EJ, Murabito JM, Rejeski WJ, Ferrucci L, Harris TB. Assessing Daily Physical Activity in Older Adults: Unraveling the Complexity of Monitors, Measures, and Methods. Journals of Gerontology Series A: Biomedical Sciences and Medical Sciences 2016;71(8):10391048; https://doi.org/10.1093/gerona/glw026

11. Lin JJ, Mamykina L, Lindtner S, Delajoux G, Strub HB. Fish'n'Steps: Encouraging Physical Activity with an Interactive Computer Game UbiComp 2006: Ubiquitous Computing; pp 261-278; https:// doi.org/10.1007/11853565_16

12. Consolvo S, McDonald DW, Toscos T, CheN MY, Froehlich J, Harrison B, Klasnja P, LaMarca A, LeGrand L, Libby R, Smith I, Landay JA. Activity sensing in the wild: a field trial of UbiFit Garden. In Proceedings of the SIGCHI conference on human factors in computing systems. ACM 2008 ; pp 17971806; https://doi.org/10.1145/1357054.1357335

13. Lane ND, Mohammod M, Lin M, Yang X, Lu H, Ali S, Doryab A, Berke E, Choudhury T, Campbell A. BeWell: A smartphone application to monitor, model and promote wellbeing. In 5th International ICST Conference on Pervasive Computing Technologies for Healthcare 2011;23-26; https://doi. org/10.4108/icst.pervasivehealth.2011.246161

14. Vichitvanichphong S, Talaei-Khoei A, Kerr D. Elderlys' Perception about The Value of Assistive Technologies for their Daily Living: Impacting Factors and Theoretical Support. Hawaii International Conference on System Sciences 2017; https://doi. org/10.24251/HICSS.2017.445

15. Sookhai L, Coppola JF, Gaur C. Intergenerational activity tracker program: Impact with health re- 
lated outcomes on older adults. Systems, Applications and Technology Conference (LISAT), IEEE Long Island 2015; pp 1-7; https://doi.org/10.1109/ LISAT.2015.7160218

16. Lebron J, Escalante K, Coppola J, Gaur C. Activity tracker technologies for older adults: Successful adoption via intergenerational telehealth. Systems, Applications and Technology Conference (LISAT), IEEE Long Island 2015; https://doi.org/10.1109/LISAT.2015.7160200

17. Burke L, Lee AH, Jancey J, Xiang L, Kerr DA, Howat PA, Hills AP, Anderson AS. Physical activity and nutrition behavioral outcomes of a home-based intervention program for seniors: a randomized controlled trial. International Journal of Behavioral Nutrition and Physical Activity 2013; https://doi. org/10.1186/1479-5868-10-14

18. Guest G, Bunce A, Johnson L. How many interviews are enough? An experiment with data saturation and variability. Field Methods 2006;18(1):24; https://doi.org/10.1177/1525822X05279903

19. Crouch M, McKenzie H. The logic of small samples in interview-based qualitative research. Social Science Information 2006;45(4):18; https://doi. org/10.1177/0539018406069584

20. Corbin J, Strauss A. Basics of Qualitative Research: Techniques and Procedures for Developing Grounded Theory (3rd ed.). Thousand Oaks, CA: Sage 2008; https://doi. org/10.4135/9781452230153

21. Bisafar FI, Parker AG. Confidence \& Control: Examining Adolescent Preferences for Technologies that Promote Wellness. In Proceedings of the 19th ACM Conference on Computer-Supported Cooperative Work \& Social Computing (CSCW '16), ACM, New York, NY, USA 2016; pp 160-171; http://dx.doi.org/10.1145/2818048.2820028

22. Stawarz K, Cox AL, Blandford A. Beyond selftracking and reminders: Designing smartphone apps that support habit formation. In Proceedings of the 33rd Annual ACM Conference on Human Factors in Computing Systems (CHI '15), ACM, New York, NY, USA 2015; pp 2653-2662; http:// dx.doi.org/10.1145/2702123.2702230

23. Schorch M, Wan L, Randall DW, Wulf V. Designing for those who are overlooked: Insider perspectives on care practices and cooperative work of elderly informal caregivers. In Proceedings of the 19th ACM Conference on Computer-Supported Cooperative Work \& Social Computing (CSCW '16), ACM, New York, NY, USA 2016; pp 787-799; http://dx.doi.org/10.1145/2818048.2819999

24. Sabelli AM, Kanda T, Hagita N. A conversational robot in an elderly care center: An ethnographic study. Proceedings of the 6 th international conference on Human-robot interaction 2011; pp 37-44; https://doi.org/10.1145/1957656.1957669

25. Olphert W, Damodaran L, Balatsoukas P, Parkinson C. Process requirements for building sustainable digital assistive technology for older people. Journal of Assistive Technologies 2009;3(3):4-13; https://doi.org/10.1108/17549450200900019

26. Laine TH, Suk HJ. Designing Mobile Augmented Reality Exergames. Games and Culture 2015;11(5):548-580; https://doi.org/ 10.1177/1555412015572006

27. Olphert W, Damodaran L, Balatsoukas P, Parkinson C. Process requirements for building sustainable digital assistive technology for older people. Journal of Assistive Technologies 2009;3(3):4-13; https://doi.org/10.1108/17549450200900019 